\title{
Actinomyces in the female genital tract
}

\section{A preliminary report}

\author{
G C GRICE* AND S HAFIZ† \\ From the *Department of Genitourinary Medicine, Royal Hallamshire Hospital, and the + Department of \\ Medical Microbiology, Medical School, Sheffield
}

SUMMARY Actinomyces spp were isolated by culture of endocervical specimens from two groups of women attending the department of genitourinary medicine of the Royal Hallamshire Hospital, Sheffield. The first group consisted of 78 users of intrauterine contraceptive devices (IUCD) of whom $20(25 \cdot 6 \%)$ were culture positive. The second group contained 63 women using various forms of contraception $12(19 \%)$ of whom were culture positive. None of these 12 women had an IUCD or foreign body in situ. The results suggest that Actinomyces spp may be part of the commensal flora of the genital tract in some women.

\section{Introduction}

Actinomyces is a normal commensal of dental caries, the tonsillar crypts, and the colon but does not seem to invade intact mucus membranes unless there is preexisting erosion or perforation of the tissue. Actinomycosis classically affects three areas of the bodythe face and neck, thorax, and abdomen. It is a chronic disease characterised by superficial or visceral granulomatous lesions which form abscesses which break down and produce multiple draining sinuses. Characteristic "sulphur granules" may be found in the exudate, the sinus walls, or the deep lesions.

There has been an increasing number of reports of actinomyces infecting the female genital tract, particularly in women using an intrauterine contraceptive device (IUCD). ${ }^{1-3}$ Several species have been identified, the most common being Actinomyces israelii, but $A$ naeslundii, $A$ eriksonii, and $A$ proprionica may also be responsible. Most studies have relied on cervical cytology or tissue histology for diagnosis because isolation of actinomyces by culture was considered less reliable, success rates of up to $36 \%$ having been reported. ${ }^{45}$ An isolation rate of $86 \%$ was obtained more recently using a culture medium which incorporated metronidazole. ${ }^{6}$

Since October 1981 we have isolated actinomyces by culture from women using either IUCDs or alternative contraceptive methods.

Address for reprints: Dr G C Grice, Department of Genttourinary Medicine, Royal Hallamshire Hospital, Glossop Road, Sheffield $\mathrm{S} 102 \mathrm{JF}$

Accepted for publication 19 April 1983

\section{Patients and methods}

STUDY POPULATION

The study initially comprised an unselected group of 78 women attending the department of genitourinary medicine who used an IUCD. A further 63 women who were part of a separate investigation were added to this study for comparison as they used various contraceptive methods.

\section{CULTURE METHODS}

A further endocervical swab was taken from each patient on completion of the normal screening investigations for sexually transmitted infection. The swabs from the 78 women using IUCDs were immediately plated on to two Petri dishes stored at room temperature; one contained brain heart infusion agar (BHIA) and the other BHIA with $0.4 \%$ sodium bicarbonate. (This modified medium is currently under investigation.) Finally the swab was placed in thioglycollate broth. These specimens were transported to the laboratory where culture was performed for a full 10 days as recommended by the Centers for Disease Control, Atlanta, Georgia, United States. ${ }^{7}$ The figure summarises the technique together with the methods used for species identification. The swabs from the 63 women using various contraceptive methods were placed in Amies' transport medium and subcultured on BHIA in the laboratory.

IDENTIFICATION METHODS

Identification of Actinomyces spp was based on the appearance of Gram stained smears and biochemical 


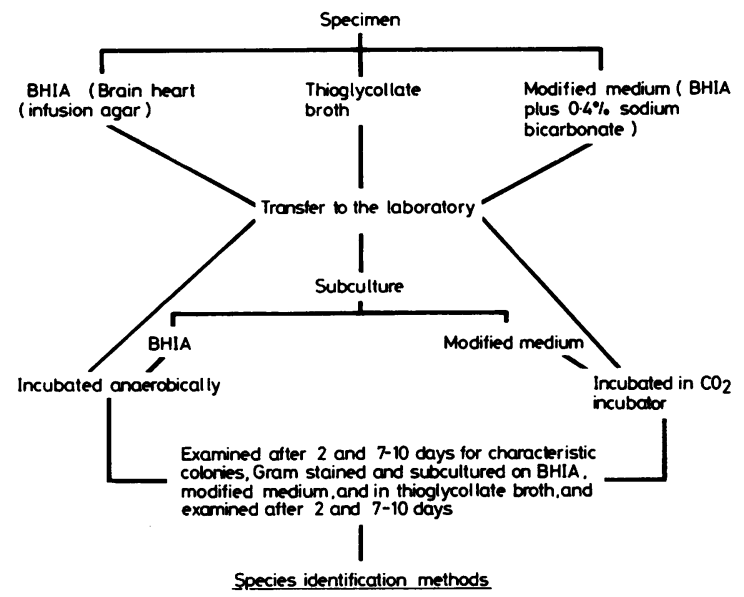

Grom staining

Catalase test

Starch hydrolysis test

Gelatin liquefaction test

Nitrate recuction

Litmus mild reaction

Carbohydrate fermentation tests

(Glucose, xylose, mannitol, ratfinose. glycerol)

FIGURE Summary of the methods used for culture of actinomyces and species identification.

tests. None of the Actinomyces species break down peroxide to produce catalase, liquefy gelatin, or produce much acid in the litmus milk test. $A$ israelii and $A$ naeslundii respond with only limited starch hydrolysis but do give a positive nitrate reduction test, whereas $A$ bovis fails to reduce nitrate but gives a pronounced response to starch. Production of acid in the sugar fermentation tests is a useful means of identifying species. All ferment glucose, but only $A$ israelii ferments xylose and mannitol and $A$ bovis only occasionally utilises glycerol. $A$ naeslundii ferments raffinose well, whereas $A$ israelii gives variable results.

\section{Results}

Actinomyces was cultured from $20(25 \cdot 6 \%)$ of the 78 women with an IUCD in situ. The type of IUCD was unknown in seven of them; 12 used a copper device and one a Saf-T-Coil. The duration of use ranged from six months to six years. None of these women had pelvic inflammatory disease (PID). Their symptoms were vaginal discharge (seven), vulval irritation (three), dysuria (one), and pleuritic pain (one); seven women were asymptomatic and one had vulval warts. Other causes for the symptoms were found in all but five of these women, who were not treated but advised to have their IUCD removed.
Of the 63 patients using various contraceptive methods $12(19 \cdot 1 \%)$ were infected with actinomyces (table). Five of the women had an IUCD in situ and all were culture negative. Actinomyces was isolated in $12(20.7 \%)$ of the 58 non-IUCD users. Only one of the 12 infected women had PID; she was currently using no contraception although an IUCD had been removed six months earlier during a similar episode of pelvic pain. The symptoms of the remaining 11 infected patients not using an IUCD were: vaginal discharge (five), vulval irritation (three), dysuria and loin pain (one) (three having more than one symptom); four were asymptomatic and one had vulval warts. Other causative pathogens were found in all but three of the symptomatic women and they were treated accordingly. The three women with no other pathogen were not treated. The species of actinomyces identified included 29 isolates of $A$ israelii, one each of $\boldsymbol{A}$ bovis and $\boldsymbol{A}$ naeslundii, and one unidentified species.

TABLE Results of actinomyces culture related to contraceptive methods in 63 unselected women

\begin{tabular}{lcc}
\hline $\begin{array}{l}\text { Method of } \\
\text { contraception }\end{array}$ & $\begin{array}{l}\text { No of } \\
\text { women }\end{array}$ & $\begin{array}{l}\text { No (\%) } \\
\text { culture } \\
\text { positive }\end{array}$ \\
\hline $\begin{array}{l}\text { Intrauterine contraceptive } \\
\text { device (IUDC) }\end{array}$ & 5 & 0 \\
$\begin{array}{l}\text { Oral contraceptive } \\
\text { Sheath }\end{array}$ & 23 & $4(17 \cdot 4)$ \\
Cap & 3 & $3(100)$ \\
Sterilisation & 1 & $1(100)$ \\
No contraception & 1 & $0(0)$ \\
Total & 28 & $4(14 \cdot 3)$ \\
\hline
\end{tabular}

\section{Discussion}

Actinomycosis has been well described and classically affects three areas (cervicofacial, abdominal, and thoracic) of the body. An increasing numer of infections of the female genital system have been reported, usually in women using an IUCD as a form of contraception or where some other foreign body (tampon or pessary) has been present. ${ }^{2}$ Orogenital contact has been suggested as a possible source of infection. ${ }^{2}$ No type of IUCD has been found to protect against infection, and serious complications associated with IUCD use include dissemination of actinomyces, hepatic abscess, intracranial abscess, ${ }^{2}$ and even death. ${ }^{8}$ Our finding of $25.6 \%$ of IUCD users with cervical actinomyces is consistent with that of other workers, who reported a figure of $25 \%$, of whom 1-7-25\% had PID. ${ }^{4} 10$ We were also able to detect actinomyces in the genital tract of $12(20 \cdot 7 \%)$ of 58 women using alternative contraceptive methods with no foreign body in situ. This is also consistent 
with a recent report of actinomyces isolation from $27 \%$ of 30 women without an IUCD or foreign body. ${ }^{11}$ The finding of actinomyces in such women suggests a commensal role in the genital tract.

Contrary to previous reports, we found that culture was a reliable method of isolating Actinomyces spp. Histological examination and cervical cytology have not yet been compared as screening methods for diagnosis. The former is impractical in clinics, and the specificity of the latter is uncertain in view of possible confusion of actinomyces with morphologically similar vaginal flora. ${ }^{11}$ Until sera for immunofluorescence tests become freely available the sensitivity of cervical cytology for screening purposes remains unknown.

Despite the small numbers so far investigated this preliminary study indicates that the presence of actinomyces in the genital system of women is not uncommon and may be found in the absence of an IUCD or foreign body. It has also shown that culture techniques may prove more successful than previously thought. Controlled studies of large numbers of women practising various methods of contraception are in progress to determine the precise role of actinomyces as a commensal in the female genitourinary tract and its relationship to contraception and sexual practice and to establish a culture technique which is reliable, fast, and economical.

We thank Hilary Gooch for her technical expertise and Dr G R Kinghorn for his advice in the preparation of this paper.

This paper was presented at the July meeting of the Pathological Society of Great Britain and Ireland.

\section{References}

1. Charnock M, Chambers TJ. Pelvic actinomycosis and intrauterine contraceptive devices. Lancet 1979; i: 1239-40.

2. Gupta PK, Erozan YS, Frost JK. Actinomycetes and the IUD: an update. Acta Cytol 1978;22:281-2.

3. Jones MC, Buschmann BD, Dowling EM, Pollock, Helen M. The prevalence of actinomycetes-like organisms found in cervico-vaginal smears of 300 IUD wearers. Acta Cytol 1979;23:282-3.

4. Bhagavan BS, Gupta PK. Genital actinomycosis and intrauterine devices. Hum Pathol 1978; 9:567-8.

5. Hagar WD, Douglas B, Majmudar B, et al. Pelvic colonisation with actinomyces in women using intrauterine contraceptive devices. Am J Obstet Gynecol 1979; 135:680-4.

6. Traynor RM, Parratt D, Duguid HLD. Isolation of actinomycetes from cervical specimens. J Clin Pathol 1981;34:914-6.

7. Ajello L, Georg LK, Kaplan W, Kaufman L. Laboratory manual for medical microbiology. Atlanta, Georgia: Department of Health, Education and Welfare, Public Health Service, Centers for Disease Control, 1966.

8. Hager WD, Majmudar B. Pelvic actinomycosis in women using intrauterine contraceptive devices. Am J Obstet Gynecol 1979; 133:61-3.

9. Sykes GS, Shelley G. Actinomyces-like structures and their association with intrauterine contraceptive devices, pelvic infection and abnormal cytology. Br J Obstet Gynaecol 1981;88:934-7.

10. Valicenti JF, Pappas AA, Graber CD. Detection and prevalence of IUD associated actinomyces colonisation and related morbidity. JAMA 1982;247:1149-52.

11. Curtis EM, Pine L. Actinomyces in the vaginas of women with and without intrauterine contraceptive devices. Am J Obstet Gynecol 1981; 140:880-4. 